\title{
GEOELECTRIC FIELD MEASUREMENTS ON A PLANETARY SCALE: OCEANOGRAPHIC AND GEOPHYSICAL APPLICATIONS
}

\author{
A.D. Chave ${ }^{1}$, D.S. Luther ${ }^{2}$, L.J. Lanzerotti ${ }^{3}$, and L.V. Medford ${ }^{3}$
}

\begin{abstract}
The characteristics of an initial year of electric field measurements obtained from a long $(\approx 3900 \mathrm{~km})$, unpowered submarine cable extending from Point Arena, California, to Hanauma Bay, Hawaii are reported. The power spectrum estimated from the cable time series is similar to that from seaflonr point electric field sensors at frequencies above $1 \mathrm{cpd}$, but differs at lower frequencies by decreasing monotonically down to at least $0.02 \mathrm{cpd}$. The multiple squared coherence between the cable data and three component geomagnetic fluctuations measured at Fresno, Ca., is high $\left(\gamma^{2} \approx 0.9\right)$ from $1.0-0.09 \mathrm{cpd}$, then declines slowly with decreasing frequency. Maps of the squared coherence between the cable voltage and the surface air pressure, wind stress, and wind stress curl derived from the Fleet Numerical Oceanography Center product over the entire eastern North Pacific show no significant relationships at frequencies above $\approx 0.05 \mathrm{cpd}$, but strong nonlocal coherence with the wind stress curl forcing obtains at lower frequencies. Taken together, these observations support a transition from high frequency dominance by external sources to low frequency dominance by oceanic motional sources at $0.05-0.1 \mathrm{cpd}$, a considerably smaller value than has previously been observed with point electric field sensors on the ocean bottom. The difference is caused by horizontal averaging of the short spatial scale motional component over the long submarine cable.
\end{abstract}

\section{Introduction}

Natural electric currents are induced in the ocean by three principal types of sources: 1) fluctuations of the magnetic field at Earth's surface caused by external electric current systems, 2) the dynamo interaction of moving, conducting seawater with Earth's stationary main magnetic field, and 3) processes in Earth's core. Source 1 has motivated application of the magnetotelluric method to studies of the solid earth beneath the oceans and investigations of ionospheric and magnetospheric phenomena using data from submarine cables. The motional electric field (source 2) gives a spatially-smoothed measure of the water velocity; at subinertial periods and for a given point on the seafloor, it is proportional to the vertically-integrated, seawater conductivity weighted water velocity averaged over a horizontal radius of a few water depths (Chave and Luther, 1990). For a submarine cable, this quantity is also integrated along its length. The DC contribution from source 3 has been examined on several occasions with

\footnotetext{
${ }^{1}$ Woods Hole Oceanographic Institution

${ }^{2}$ Scripps Institution of Oceanography

${ }^{3}$ AT\&T Bell Laboratories
}

Copyright 1992 by the American Geophysical Union.

Paper number 92GL01286

0094-8534/92/92GL-01286\$03.00 results that appear to be location dependent (e.g., Lanzerotti et al., 1992).

Any source may dominate the oceanic electric field over different parts of the spectrum, depending on the relative source magnitudes, the spatial scale of the excitation, and the response characteristics of the sensor. Based on observations from regions of weak eddy variability, the seafloor point electric field is known to be dominated by external sources at periods shorter than a few days, but motional sources predominate at longer periods (e.g., Luther et al., 1991). While the spatial scales associated with some external sources can approach planetary size $(>1000 \mathrm{~km})$, the scales of motional sources are those of the hydrodynamic flows and hence typically smaller. As a result, it is expected that an electric field measurement from a long submarine cable will display different properties than for a point sensor because horizontal integration will reduce the motional component. This might be of some importance because motional induction produces the background against which both core and external fields are measured.

In this paper, the initial year of an ongoing program of electric potential variation measurements from a pair of abandoned submarine cables extending from California to Hawaii is reported. These data series are of considerably longer duration than those published in earlier studies over basin-scale distances.

\section{Data}

The now-decommissioned Hawaii-1 (HAW-1) system consists of a pair of parallel cables separated north-south by $\approx 120 \mathrm{~km}$ and stretching from Point Arena, Califomia $\left(38^{\circ} 59^{\prime} \mathrm{N}, 123^{\circ} 42^{\prime} \mathrm{W}\right)$ to Hanauma Bay, Hawaii $\left(21^{\circ} 16^{\prime} \mathrm{N}\right.$, $157^{\circ} 42^{\prime} \mathrm{W}$ ). The length of each of the cables is $\approx 3900 \mathrm{~km}$. Details on the measuring system may be found in Lanzerotti et al. (1992).

The cable time series used in this study consist of 20 minute values from 7 April 1990 through 10 April 1991. The data sets are continuous except for two short gaps of $\approx 3.6$ and $\approx 0.2 \mathrm{~d}$ duration which occurred in July and September 1990 respectively. Hourly mean values of the three geomagnetic field components were obtained for calendar year 1990 from the Fresno, Boulder, and Honolulu observatories operated by the U.S. Geological Survey. These time series contained data gaps of up to several days duration constituting less than $2 \%$ of the data for Fresno and Boulder and over $10 \%$ at Honolulu. The Fleet Numerical Oceanography Center (FNOC) weather product consisting of objectively-analyzed estimates of the surface air pressure and wind velocity at $6 \mathrm{~h}$ intervals was obtained for 513 sites separated by $\approx 300 \mathrm{~km}$ covering the entire easterr North Pacific for March 1990 through April 1991. The wind data were converted to wind stress and its curl using methods described in Chave et al. (1991).

Both the cable electric field and observatory magnetic field data are dominated by the periodic variations due to 
the solar daily variation $\left(S_{q}\right)$ and its harmonics and the ocean tides. While these are of no direct relevance to the present study, they constitute a complication for further analysis. Estimates of the complex amplitudes of sinusoids at $24,12,8,6,4.8$, and $4 \mathrm{~h}$ periods as well as the four largest ocean tides and the record mean were made by robust least squares. Because the solar daily variation displays marked seasonal variability, up to five additional sidebands on either side of each $\mathrm{S}_{\mathrm{q}}$ center frequency separated by integral multiples of $1 \mathrm{cpy}$ were also used. The resulting periodic signal was removed from the data.

Since the behavior of the geoelectric data at the longest resolvable periods is of immediate interest, considerable attention was paid to filling the data gaps in the cable and magnetic observatory data in a physically-meaningful manner. This started with the computation of a multivariate frequency-domain transfer function between each gappy data component and all three data series at a reference geomagnetic observatory using a robust method, yielding both the transfer function complex amplitude and its jackknife uncertainty at frequencies spaced at equal logarithmic intervals. In principle, the impulse response computed from the transfer function could be used to fill the gaps by convolution, assuming that there are not coincident data gaps at the reference station. However, the transfer function estimates are both incomplete and imperfect, and it is not possible to directly transform the transfer function to the time domain impulse response. Instead, a regularization procedure was applied in which a functional of the impulse response is minimized subject to fitting the transfer function and its error at some specified level of misfit, in a similar manner to that described by Egbert (1992). The functional is chosen to minimize the roughness of the Fourier transform of the impulse response, and the result is not overly sensitive to the details of the either the constraint or the misfit.

The regularized impulse response was first used to complete the Fresno time series using Boulder as a reference. An identical procedure was then employed to fill the gaps in the cable time series from the corrected Fresno observatory data. Finally, the more extensive data gaps in the Honolulu observatory data were corrected using the Fresno observatory data. None of the conclusions presented below depend on the details of this procedure. However, it should be remembered that this approach presumes that all of the processes measured by the cable are predictable from magnetic field observations. Given the small fraction of cable data which have to be corrected, this is unlikely to lead to major errors.

\section{Results and Discussion}

Figure 1 compares multiple window power spectra computed from hourly means of (bottom) the north cable data and (top) seafloor electric field time series from point sensors placed beneath the Gulf Stream near $37^{\circ} 20^{\prime} \mathrm{N}$, $67^{\circ} 40^{\prime} \mathrm{W}$ (solid line) and in a weak eddy variability part of the North Pacific at $40^{\circ} 37^{\prime} \mathrm{N}, 167^{\circ} 41^{\prime} \mathrm{W}$ (dashed line). The point sensor time series were de-tided in an identical manner to the cable data. All of the spectra show a slow decrease in power with frequency above $1 \mathrm{cpd}$ and have
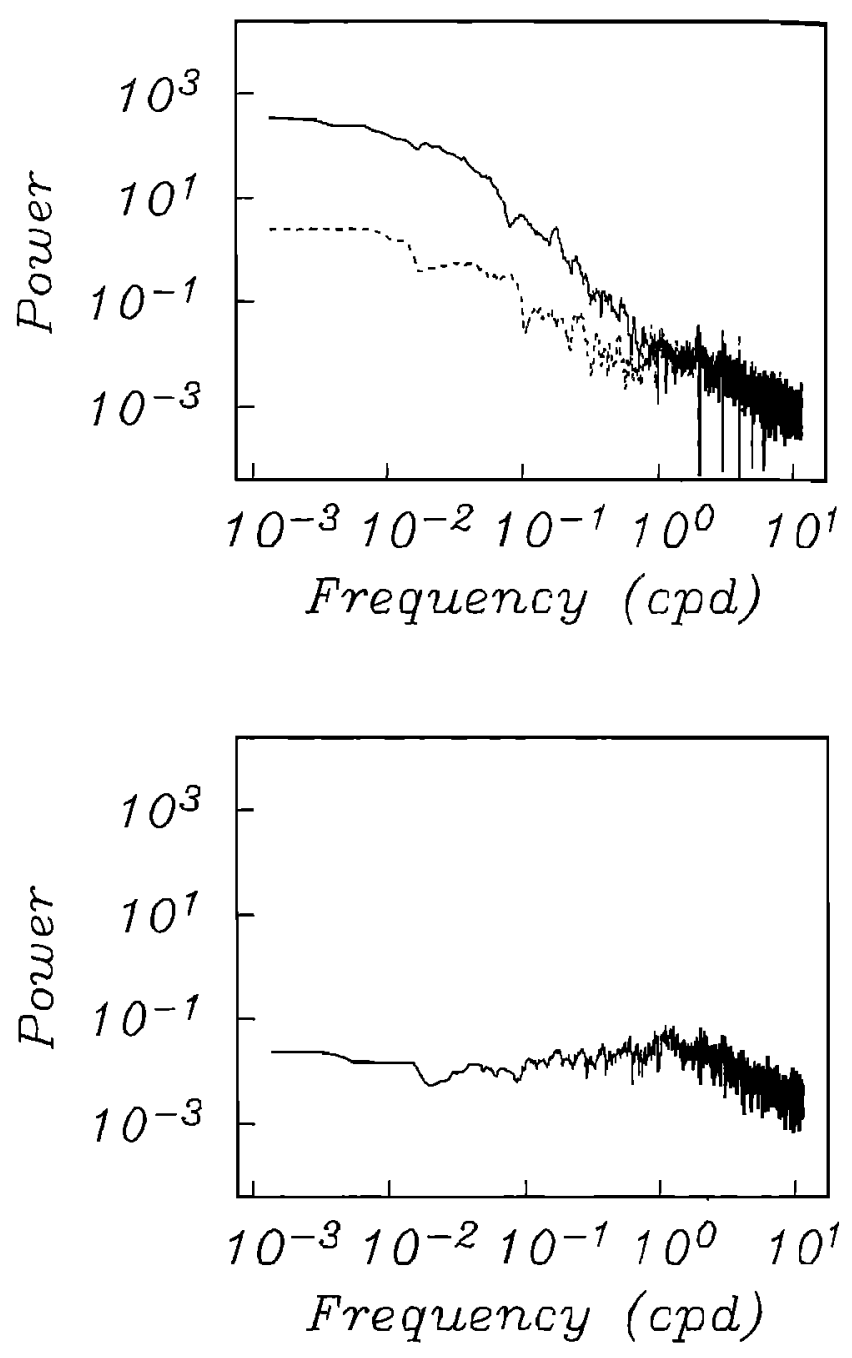

Fig. 1. Power spectra (in $\mathrm{mV}^{2} \mathrm{~km}^{-2}$ per cpd) of hourly means of the electric field from the north segment of HAW-1 for the time period April 1990 to April 1991 (bottom) and for two year-long time series of the north electric field collected with point sensors (top). For the latter, the solid line is from a site located beneath the Gulf Stream, while the dashed line is from a weak eddy variability region in the North Pacific; see text for details. The estimates have a resolution bandwidth of $\approx 0.02 \mathrm{cpd}$ at all frequencies and possess $\approx 14$ degrees of freedom, yielding a $95 \%$ confidence limit of $(0.54$, 2.49) times the power at each frequency.

similar spectral slopes, although the magnitude of the cable spectrum is about a factor of two larger. The small magnitude discrepancy is probably due to orientation differences between the cable and the point sensor. However, large differences in spectral behavior are apparent at frequencies below $\approx 1 \mathrm{cpd}$. Both of the point sensor spectra rise rapidly as frequency decreases, with a steeper slope and larger magnitude for the Gulf Stream data. The low frequency power is due to motional induction, and the $\approx 2$ decade difference in power between the Gulf Stream and Pacific data at the lowest frequencies is consistent with the known oceanic energy levels. A low frequency increase in power 
is completely absent for the cable data, suggesting that significant horizontal averaging of the short (compared to the cable length) scale motional electric fields has occurred.

There is no evidence for a minimum in power for the cable data at low frequencies to within the frequency resolution of the estimate. Note that the record mean of 0.200 $\mathrm{mV} / \mathrm{km}$ (with the sign such that current in the cable is flowing from Hawaii to North America) has been removed prior to computing the estimate in Figure 1 (bottom); integrating the spectrum over its resolution bandwidth $(\approx 0.02 \mathrm{cpd})$ centered on zero frequency and computing an rms amplitude for the lowest resolvable frequencies yields $0.012 \mathrm{mV} / \mathrm{km}$, which is more than an order of magnitude below the record mean. These values are similar to those discussed by Lanzerotti et al. (1992). However, the true mean typically cannot be resolved from components with frequencies of order one over the record length by least squares methods, and hence it is not possible to separate DC electric fields from possible large annual or interannual fluctuations induced by the ocean. A longer record will be required to improve on this bound.

Figure 2 shows the multiple squared coherence between the electric field on the north cable and all three magnetic field components from the geomagnetic observatory at Fresno, California. The estimate is well above the zero coherence level at $95 \%$ significance at all save the highest resolvable frequencies. The decrease in coherence above 1 cpd as well as the increase in variability is due to nonstationarity of the external geomagnetic field. The most interesting feature of Figure 2 is the high coherence value $\left(\gamma^{2} \approx 0.9\right)$ from $\approx 1 \mathrm{cpd}$ down to $\approx 0.09 \mathrm{cpd}(11 \mathrm{~d}$ period); the coherence decreases slowly below this frequency to the lowest resolvable value of $0.022 \mathrm{cpd}$, but is still statistically meaningful. The coherence at the lowest frequencies could

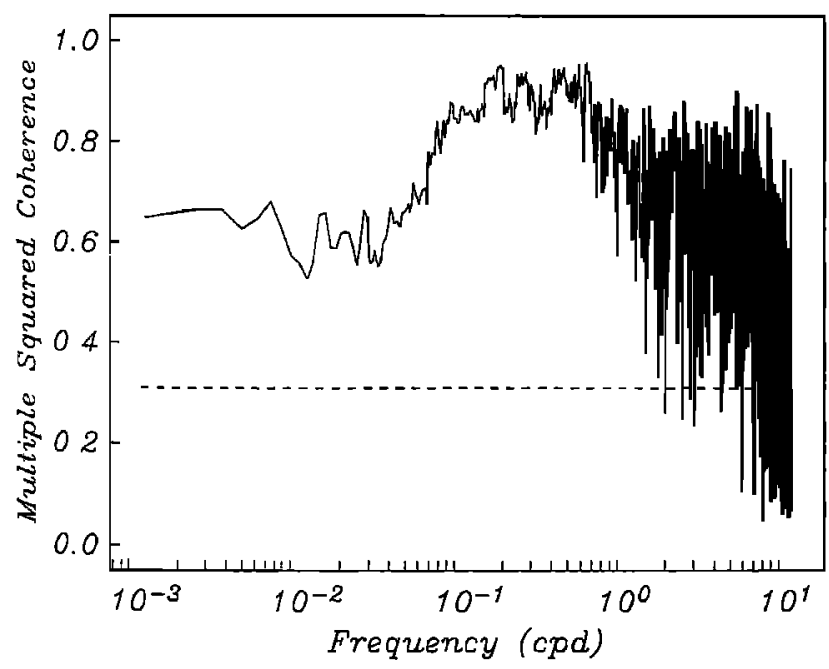

Fig. 2. Multiple squared coherence between the voltage on the north segment of HAW-1 and all three geomagnetic field components from Fresno, California for the time period April through December 1990. The estimate has a resolution bandwidth of $\approx 0.044 \mathrm{cpd}$ and possesses $\approx 18$ degrees of freedom at each frequency. The dashed line shows the approximate zero coherence level at $95 \%$ significance. be due to similarity of long term trends in the two data sets, and should not be emphasized. Since motional magnetic fields have not been observed except at the ocean tidal frequencies, the high coherence seen in Figure 2 suggests that the electric field measured by the HAW-1 cable is dominated by external sources out to periods of order two weeks. This should be contrasted with point electric field measurements which typically are incoherent with the magnetic field at periods in excess of 2-4 d even in regions of weak eddy variability. A similar coherence estimate obtains when Honolulu observatory is substituted for Fresno.

To further elucidate the low frequency source mechanism, maps of the squared coherence between the cable data and the atmospheric variables (surface air pressure, wind stress, and wind stress curl) were computed for 15 period bands between 4 and 68 days in an identical manner to that used by Chave et al. (1992). No significant coherence with wind stress curl was observed at periods shorter than $20 \mathrm{~d}$, in contrast to point electric field measurements which are coherent to about $4 \mathrm{~d}$ period. However, extended patches of very high $\left(\gamma^{2}>0.7\right)$ coherence are seen at periods longer than $20 \mathrm{~d}$, as shown in Figure 3 for a band centered on 25 d. Here a region of very strong coherence is located to the south of the cable; the peak squared coherence is 0.76 at zonal grid point 17 , meridional grid point 11 . The coherence is typically highest for the wind stress curl at all periods over $20 \mathrm{~d}$ as compared to the remaining atmos-

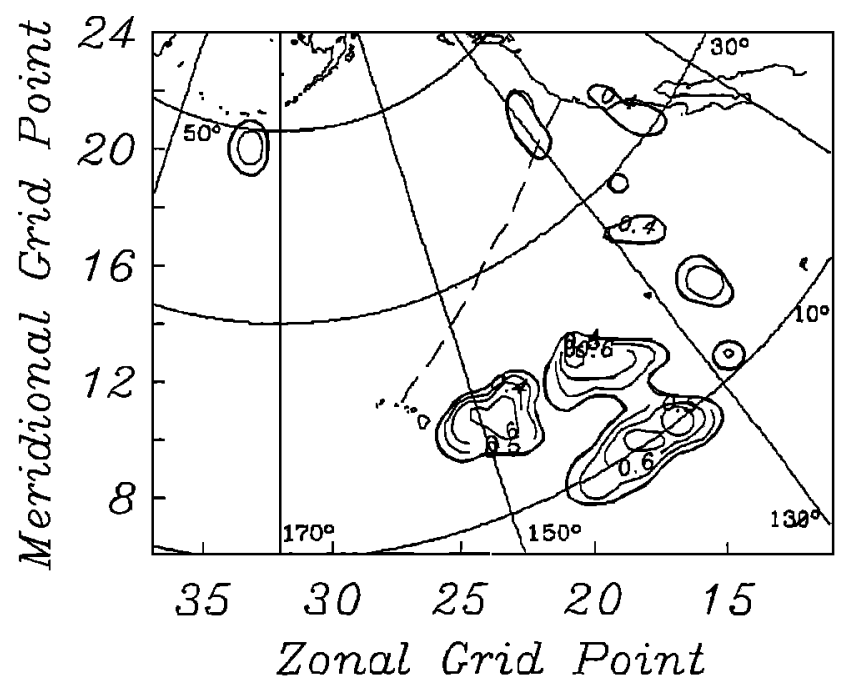

Fig. 3. Map of the squared coherence at 25 (19-36) $d$ period between the voltage on the north segment of HAW-1 and the wind stress curl at 425 points over the North Pacific computed from the FNOC product. The results are shown on a polar stereographic projection with selected latitude and longitude contours and the outlines of major land features to guide the eye. The zonal and meridional grid points are those on which the FNOC product is provided. Only coherence values which exceed the zero level at $95 \%$ significance of 0.39 were included in the contouring process; these are shown at steps of 0.1 beginning at 0.4 . The dashed line shows the track of the cable. 
pheric variables, probably due to a better wavenumber match to the integrated velocity measured by the cable and despite the expected lower noise level in the surface air pressure.

Thus, Figures 2 and 3 strongly suggest that the extended electric field measurement derived from a seafloor cable is dominated by external sources at periods of 10-20 days or less and strongly influenced by motional induction at longer periods. This serves as a dramatic illustration of spatial averaging.

These measurements suggest two possible applications for electric field measurements using abandoned submarine cables. First, seafloor magnetotelluric studies based on point electric field measurements are typically not feasible at periods longer than 1-2 d due to motional induction noise. The spatial averaging of the motional component on a long cable can extend this limit to periods of about two weeks. This would increase the depth of resolution well into the lower mantle. Geophysical studies based on very low frequency electric field time series are almost unprecedented, and none of the extant data are taken in oceanic regions. At the same time, the relative weakness of motional fields suggests that submarine cables offer inherent advantages over point sensors for dynamo studies.

Second, to lowest order the HAW-1 cable crosses half of the North Pacific gyre. While it is tempting to ascribe transport interpretations to the low frequency electric field fluctuations on the cable, there are numerous complications which make this risky without more extensive checking. This does not preclude using the cable voltage as an index to watch for relative changes in the circulation of the gyre. This is especially attractive because very long (years to decades) time series can in principle be collected on some submarine cables at very low cost, especially when compared to more conventional oceanographic techniques. However, the feasibility of oceanic monitoring must be further verified by examining the very long period coherence of the cable voltage with the geomagnetic field to ascertain that external fields are sufficiently weak. This will require time series of several years duration.

Acknowledgements. It is a pleasure to acknowledge the technical assistance of J.S. Kraus, C.H. Sayres, and C.G. Maclennan of Bell Laboratories and J. Bytof of SIO. We wish to thank many AT\&T personnel, including J.M. Barrett, J.E. Rogalski, T.E. Wills, A. Ong, J. McNeely, R. Spon, D. Sitts, R. Thompson, E. Wells, and K. Wiggins, for making these measurements possible. This work was supported by NSF grant OCE91-96236 at WHOI and NOAA grant NA16RC0545-01 at SIO. This is WHOI contribution 8017.

\section{References}

Chave, A.D., and D.S. Luther, Low-frequency, motionally induced electromagnetic fields in the ocean, 1. Theory, J. Geophys. Res.. 94, 7185-7200, 1990.

Chave, A.D., D.S. Luther, and J.H. Filloux, Variability of the wind stress curl over the eastern North Pacific: implications for the oceanic response, J. Geophys. Res. 96, 18361-18379, 1991.

Chave, A.D., D.S. Luther, and J.H. Filloux, The Barotropic Electromagnetic and Pressure Experiment 1. Atmospherically-forced barotropic currents, $\underline{\mathrm{J}}$ Geophys. Res. 97, in press, 1992.

Egbert, G.D., Non-causality of the discrete-time magnetotelluric impulse response, Geophysics, in press, 1992.

Lanzerotti, L.J., C.H. Sayres, L.V. Medford, J.S. Kraus, and C.G. Maclennan, Earth potential over $4000 \mathrm{~km}$ between Hawaii and California, Geophys. Res. Lett.. 11 . 1177-1180, 1992.

Luther, D.S., J.H. Filloux, and A.D. Chave, Low-frequency, motionally induced electromagnetic fields in the ocean, 2, Electric field and Eulerian current comparison from BEMPEX, I. Geophys. Res. 96, 12797-12814, 1991.

A.D. Chave, Woods Hole Oceanographic Institution, Woods Hole, MA 02543

L.J. Lanzerotti, AT\&T Bell Laboratories, 600 Mountain Ave., Murray Hill, NJ 07974

D.S. Luther, Scripps Institution of Oceanography, 0230, La Jolla, CA 92093

L.V. Medford, AT\&T Bell Laboratories, 600 Mountain Ave., Murray Hill, NJ 07974

(Received April 7, 1992;

revised May 21, 1992;

accepted May 21, 1992) 